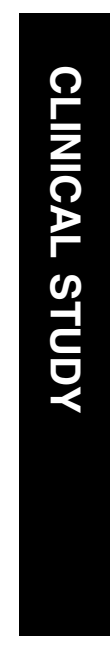

\title{
Persistency with medical treatment for glaucoma and ocular hypertension in the United Kingdom: 1994-2005
}

'Division of Community Health Sciences, St George's, University of London, Cranmer Terrace, London, UK

${ }^{2}$ Moorfields Eye Hospital, London, UK

Correspondence: CG Owen, Division of Community Health Sciences, St George's,

University of London, Cranmer Terrace, Tooting, London SW17 ORE, UK Tel: + 44020 87252796; Fax: + 4402087253584 E-mail: cowen@sgul.ac.uk

Received: 21 February 2008 Accepted in revised form: 10 June 2008

Published online: 11 July 2008

Competing interests: RW is a member of one of the Pfizer's advisory committees, and has received travel grants and honoraria from Pfizer for chairing a meeting of expert opinion concerning the treatment of glaucoma. RW has conducted research indirectly funded by grants to IGA from Pharmacia (taken over by Pfizer) for research into the adverse effects of topical beta blockers

\section{Abstract}

Purpose To examine trends and demographic factors affecting persistence with ocular hypotensive therapy, from a period before prostaglandins were available to when they were the most common therapy.

Methods Computerised patient records from 94 general practices across the Unitd Kingdom, identified 5670 registered patients newly prescribed an ocular hypotensive drug (1993-2005). Persistence was defined as continuing therapy without a 90-day gap in prescription for (i) any ocular hypotensive and (ii) initial monotherapy. Time to failure with the treatment was compared using proportional hazard analyses, adjusted for age, gender, practice, year of initial treatment, and a sociodemographic indicator. Study findings were set in the context of a review of the literature.

Results Percentage persistent at 1-year rose after 1997 when prostaglandins were introduced; from $61 \%$ in $1994-1996$ to $70 \%$ in 2002-2004. Persistence with any treatment did not differ between those initiated on $\beta$-blockers compared to prostaglandins (1.05, 95\% CI 0.93-1.17). However, $20 \%$ of subjects initiated on $\beta$-blockers received a prostaglandin by 1 year. Conversely, $8 \%$ of those initiated on prostaglandins received a $\beta$-blocker. When failure with initial therapy was considered, $\beta$-blockers appeared worse (1.35, 95\% CI 1.21-1.50); this was consistent with findings from six studies in the review (1.40, 95\% CI 1.34-1.46). Neither gender nor social factors were associated with persistence, but younger subjects (35-64 years) were
CG Owen'1, IM Carey', S de Wilde1, PH Whincup'1, R Wormald ${ }^{2}$ and DG Cook ${ }^{1}$

significantly more likely to fail as were those over 85 years.

Conclusions Introduction of prostaglandins may explain an improvement in persistence over a decade. However, whether the higher cost of initiating patients on prostaglandins is justified remains questionable unless clinically indicated.

Eye (2009) 23, 1098-1110; doi:10.1038/eye.2008.213; published online 11 July 2008

Keywords: treatment for glaucoma; compliance; persistence

Introduction

Glaucoma is the most common cause of irreversible vision loss throughout the world and represents a major public health burden. ${ }^{1-3}$ Management of patients with glaucoma aims to reduce intraocular pressure (IOP), either surgically and/or with medications, to halt progression of the disease. ${ }^{4}$ Management usually involves long-term use of topical medications. Benefits of treatment can only be achieved in those who comply and persist with the recommended treatment schedules. ${ }^{5}$

However, glaucoma is a life-long condition that is often asymptomatic in early stages, ${ }^{6}$ which does not lend itself to high levels of compliance with treatment. A recent systematic review concluded that less than a quarter of patients with glaucoma persist with treatment beyond 1 year. ${ }^{7}$ Patient compliance and persistence with treatment for glaucoma may be influenced by a myriad of factors including, sociodemographic characteristics, type and dosage of the treatment regimen. Potential or perceived side effects 
associated with a given treatment may also influence persistence. $^{8-10}$

Compliance with medical treatment for glaucoma and ocular hypertension is often measured using information ascertained directly from the patient (either by questionnaire or interview); such measures are subject to recall ${ }^{11,12}$ and selection bias. ${ }^{7}$ These problems can be overcome by using the electronic eye drop medication monitors. ${ }^{13,14}$ However, this method is not conducive to study large number of patients. Electronic healthcare databases offer an alternative approach, by looking at all prescriptions issued thus avoiding non-response bias. ${ }^{15-18}$ A few of previous studies using this approach have suggested that persistence with topical antihypertensive therapy for glaucoma is better for prostaglandins than for $\beta$-blockers. ${ }^{16-18}$ However, there has been a lack of consistency in the comparisons reported and in particular in the definition of persistence. A key problem with looking at persistence with initial therapy ( $\beta$-blockers or prostaglandins) at a time when a new drug is being actively promoted is gauging the degree to which switches from the old to the new drug reflect fashion rather than clinical benefit. It is possible to overcome this problem by comparing trends over time in persistence with any ocular hypotensive therapy from a period before prostaglandins being available to the present when prostaglandins are commonly used.

We therefore (i) analysed data from a primary care/ family practice computerised healthcare database (DIN-LINK, the United Kingdom) to examine trends in persistence over calendar years from a period before prostaglandins were available to a period when they were the mostly used therapy and (ii) analysed the DINLINK data to compare persistence with therapy in cohorts of subjects initiated on $\beta$-blocker or prostaglandin therapy. For (i), we defined treatment failure as (a) a period of 90 days without a prescription for any topical antihypertensive and (b) as a period of 90 days without a prescription for the initial therapy; for (ii), definition (a) was used to give a more balanced measure of persistence. In addition, to set the findings in context, we carried out a review to identify all studies that used computerised healthcare databases to compare persistence with $\beta$-blockers and prostaglandins.

\section{Methods}

\section{The DIN-LINK database}

DIN-LINK is an ongoing anonymised computerised UK database of individual primary care records accumulated since 1989, from general practices using iSOFT (formerly Torex) computer software. The database has been used previously to study trends in the prevalence of treatment for glaucoma and ocular hypertension, from 1994 to 2003. ${ }^{19}$ This report is based on 94 practices who opted to use the DIN-LINK system, which have continuous high quality data recorded from 1993 to $2005 .{ }^{20}$ We have outlined methodology for identifying good quality data in DIN-LINK, ${ }^{20}$ based on a series of indicators that assessed completeness of registration and deregistration data, and looked for consistent monthly volumes of diagnostic and prescription data, excluding periods, which failed to meet our criteria. The completeness and accuracy of DIN-LINK data have been demonstrated, by comparisons with other national data sources. ${ }^{20-22}$ The practices and GPs in DIN are as comparable to the UK norm as the practices and GPs in other GP research databases.

Morbidity and drug data are coded using computerised diagnostic and treatment codes (so called 'Read codes'). Subject's postcodes in the database are associated with a sociodemographic indicator at a small area level - the ACORN (a classification of residential neighbourhoods) index. ${ }^{23}$ The ACORN index is a commercially available socioeconomic numerical score derived using over a 100 variables from the 2001 decennial population Census. ${ }^{23}$ The index provides many levels of detail, but at its most aggregated level categorises residential neighbourhoods into five groups, ranging from 'wealthy achievers' to 'hard pressed'. ACORN data were missing for seven practices $(7 \%)$ due to technical problems with the linkage and unrelated to the type of practice. Practices were also classified by their geographic region.

\section{Patient selection}

We sought to identify patients who were started on treatment for glaucoma or ocular hypertension between 1994 and 2004, requiring that they be fully registered for a year without any previous ocular hypotensive therapy. Patients who had not been registered for a year before therapy were excluded, because it was not possible to rule out a previous history of ocular hypotensive treatment. Treatments for glaucoma and ocular hypertension were divided into seven groups, as described previously; ${ }^{19}$ (i) $\beta$-blockers, (ii) prostaglandins, (iii) cholinergic agents, (iv) sympathomimetics, (v) carbonic anhydrase inhibitors (both systemic and topical preparations), (vi) carbonic anhydrase inhibitors and $\beta$-blokers (ie, Cosopt ${ }^{\circledR}$ ), and (vii) prostaglandins and $\beta$-blockers (ie, Xalacom ${ }^{\circledR}$ ). Patients who were initially prescribed $\beta$-blockers only (i), prostaglandins only (ii), and combination therapies (defined as more than one group of treatment, group (vi) or (vii)) were identified.

Closer inspection of a random subset of the medical records revealed that $\beta$-blockers were used in preference 
to prostaglandins (and other therapies recently licensed for initial use), for short-term treatment after ocular surgery and/or when treating ocular inflammation. This makes any direct comparison of continuity of medication between therapies difficult because of the selection of those initiated on the different therapies. To address this problem, we excluded those $(n=452)$ initiated on ocular hypotensive therapy within 90 days of a topical corticosteroids and/or perioperative medication (a list of medications is available from the authors); patients were not excluded if a diagnostic code of glaucoma was entered during this period. This left 5670 registered patients initially started on therapy between 1994 and 2004.

\section{Defining time to failure}

Time to failure was defined as 90 days without a prescription for any treatment for glaucoma. Patients were not counted as a failure if they were transferred onto combination therapies, which may or may not include initial monotherapy. To assess the impact of treatment switching, we used a second definition of failure, defined as 90 days without the initial monotherapy. Follow-up was censored at 1080 days for both definitions, as failure rates appeared to plateau beyond this period (data not presented). In accordance with earlier studies, ${ }^{24}$ failures were also examined based on 60 and 120 day cutoffs, but this made little difference to the findings. Hence, a cutoff midway between these values (ie, 90 days) was chosen for the analyses.

\section{Statistical analysis}

Time to failure was assessed using proportional hazard models ${ }^{25}$ using PROC PHREG regression command in SAS version 9.1. for Solaris (SAS Institute, Cary, NC, USA). The hazard ratios of treatment failure by the type of treatment, gender, age group (35-64, 65-74, 75-84, and $\geqslant 85$ years), ACORN index (comparing those classified as 'wealthy achievers', 'urban prosperity', 'comfortably off', 'moderate means', 'hard pressed'), and year of initial treatment (in time periods 1994-1996, 1997-2001, 2002 onwards, and from years 1994-2004) were examined. Analyses were mutually adjusted for all exposures (except the exposure of interest) and additionally for practice (fitted as a fixed effect to allow for between practice consulting behaviour, differences in recording of data, and geographical location).

\section{Other studies examining persistence}

Studies using computerised healthcare databases to compare treatment failure with $\beta$-blockers $v s$ prostaglandins were identified from citations given in a recent review ${ }^{7}$ and by searching for references that cite papers that consider persistence (using Web of Knowledge cited reference search). Hazard ratios of failure (based on proportional hazard models) were extracted along with other study details (including population studied, time period, and inclusion and exclusion criteria). Failure in these other studies was defined in two ways - (i) the initial treatment being stopped (the equivalent of our '90 days without monotherapy definition') and (ii) the initial treatment being changed or added to. Some studies used a combination of (i) and (ii) in their definition, taking the earliest date as the point of failure.

Unlike other studies, we used a definition of failure that included discontinuation of all treatments. Adjusted hazard ratios (where available) were pooled by definition of failure, using the fixed-effects models throughout; these models reflect that only the random error within each study are more conservative when results of smaller studies show stronger associations and make no assumptions about the representativeness of the available studies. Results from different studies were compared to see whether they were similar using $\chi^{2}$-tests. ${ }^{26}$ Funnel plots, plotting the effect size for a given study by the number of participants, were used to assess whether small studies yield larger effect estimates compared to larger studies (so called 'small study bias' or 'publication bias'). ${ }^{27,28}$ Begg and Egger tests for funnel plot asymmetry and small study bias were also performed throughout. ${ }^{27,29}$ Although the potential sources of difference in results across studies were examined (such as the inclusion of a glaucoma diagnosis), there was insufficient statistical power to formally test whether a study characteristic was important, and hence, these are not reported.

\section{Results}

From 1994 to 2004, 5670 patients were initially prescribed treatments for glaucoma or ocular hypertension, including $\beta$-blocker only $(n=3504)$, prostaglandin only $(n=1798)$, and combination therapies $(n=368) ; 4408$ $(78 \%)$ of these had a diagnostic code for glaucoma in their record. Prostaglandins were not available before 1997 after which they rapidly gained in popularity as the treatment for initiating subjects onto therapy, so that in $2004,68 \%$ of patients were started on prostaglandins (Figure 1). We can also see that from 1997 significant numbers of subjects initiated on $\beta$-blockers received prostaglandins by 1 year; the percentage appears to have stabilised at just over 20\% (blue line, Figure 1). Conversely, from 1998 onwards, the percentage of subjects initiated on prostaglandins who received a 


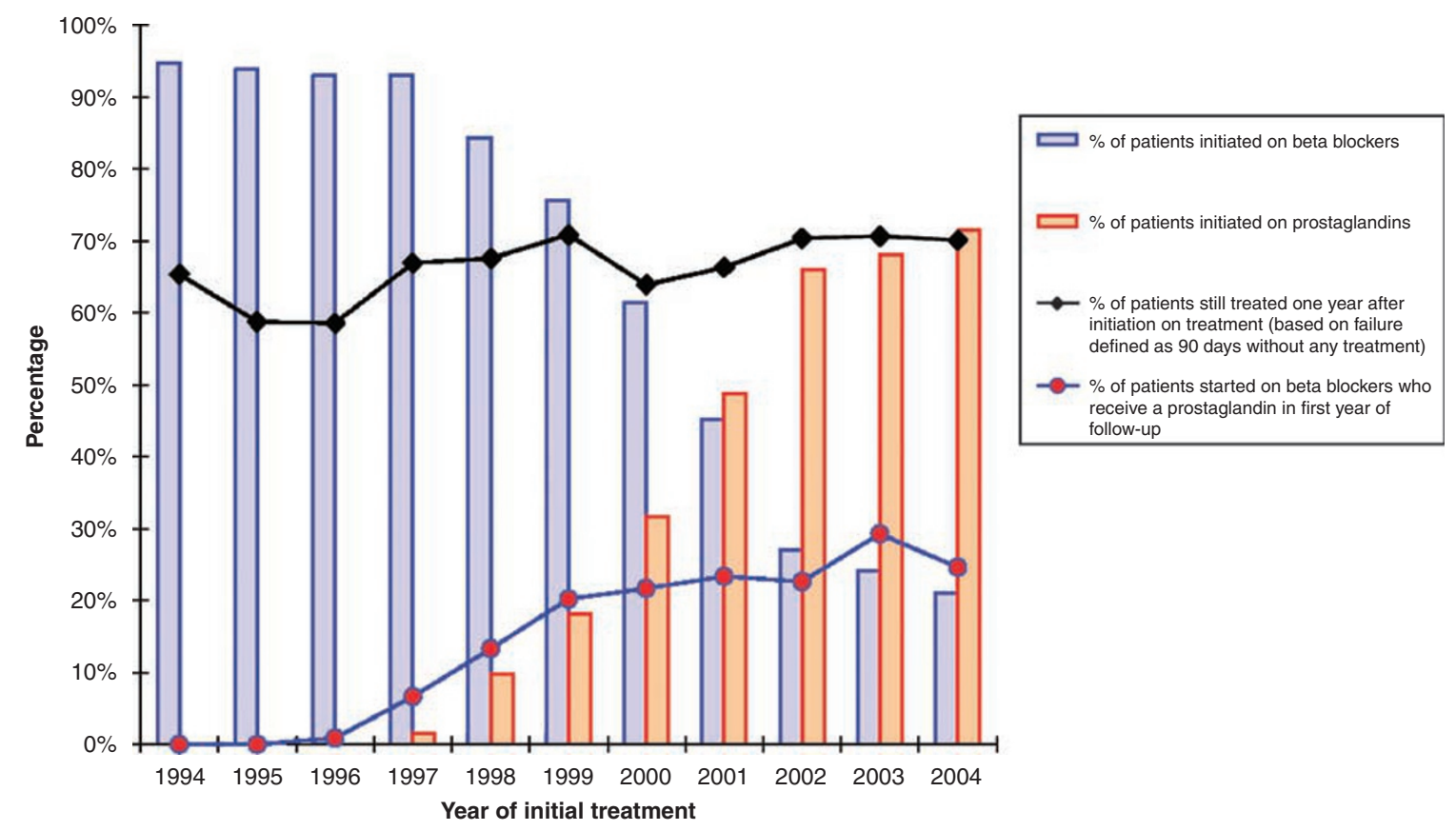

Figure 1 Glaucoma treatment patterns 1994-2004.

$\beta$-blocker by 1 year has remained stable at approximately $8 \%$ (data not presented). A similar proportion of men and women were prescribed the different treatments throughout (Table 1). The mean age of those treated with prostaglandins (from 72.5 to 75 years) appeared older than those treated with $\beta$-blockers (68-72.7 years, Table 1). There was no clear pattern with age in the minority who received a variety of combination therapies (data not presented).

Defining persistence as continuing therapy without a 90-day gap in prescription for any ocular hypotensive therapy, the percentage persistent at 1 year appeared to rise after 1997 when prostaglandins were introduced. Using survival analysis to model, the risk of failure we took 1994-1996 as the baseline when switching to prostaglandins was not an option for at least 1 year. Compared to this baseline, persistence rose from $61 \%$ at 1 year in $1994-1996$, to $67 \%$ in $1997-2001$, to $70 \%$ in 2002 2004, a one-third increase (hazard ratio for 2002-2004 vs $1994-1996=1.33,95 \%$ CI 1.22, 1.47, after adjustment for age, practice). The hazard ratios appeared to be consistent over different lengths of follow-up (data not presented).

Comparing the open and closed squares in Figure 2, we can see that, between 1998 and 2004, persistence with any treatment did not differ markedly between those initiated on $\beta$-blockers and those initiated on prostaglandins. The hazard ratio for $\beta$-blockers vs prostaglandins was 1.05 (95\% CI 0.93, 1.17). However, it must be remembered that $>20 \%$ of subjects initiated on $\beta$-blockers received a prostaglandin by 1 year (Figure 1 ). It is, therefore, no surprise that when we use a definition of failure of 90 days without a prescription for the initial therapy, $\beta$-blockers do worse (hazard ratio $=1.35,95 \% \mathrm{CI}$ $1.21,1.50)$.

Neither gender nor social factors were associated with persistence of therapy, but younger subjects (35-64 years) were significantly more likely to fail as were those over 85 years, compared to those aged $75-84$ years (Table 2 ). Restricting the comparison to only those with a glaucoma code in their record did not markedly alter the effect estimates (data not shown).

\section{Review of other studies}

In total, eight studies were identified that used healthcare databases and proportional hazard models to compare treatment failure with $\beta$-blockers and prostaglandins (Table 3). One of the studies ${ }^{16}$ included patients with a glaucoma diagnosis, who were also included in a larger population of patients treated for ocular hypertension. ${ }^{18}$ Because the hazard ratios were similar between the two studies, data from the larger population was used preferentially. There was considerable heterogeneity in the definitions of failure used (Table 3). Of the seven distinct studies, six included definitions of failure based on therapy discontinuation alone, while five also included treatment additions or changes as failure as well (Table 3). Failure to persist on $\beta$-blockers (assessed by therapy discontinuation alone) was raised in all six 
Table 1 Treatment persistence (failure defined as 90 days without any therapy) at 1 and 2 years for treatment cohorts 1994-2004

\begin{tabular}{|c|c|c|c|c|c|c|c|c|c|}
\hline \multirow{2}{*}{$\begin{array}{l}\text { Treatment cohort } \\
\text { (Year of initial treatment) }\end{array}$} & \multirow[t]{2}{*}{$n$} & \multirow[t]{2}{*}{ Mean age } & \multirow{2}{*}{$\begin{array}{c}\text { Male } \\
\text { percentage }\end{array}$} & \multicolumn{3}{|c|}{1 year follow-up } & \multicolumn{3}{|c|}{2 years follow-up } \\
\hline & & & & $N_{1}$ & Fail $^{a}$ & $\%$ trt & $\mathrm{N}_{2}$ & $\mathrm{Fail}^{\mathrm{a}}$ & $\%$ trt \\
\hline \multicolumn{10}{|l|}{$\beta$-blockers } \\
\hline 1994 & 423 & 71.4 & 52 & 401 & 136 & 66 & 505 & 224 & 56 \\
\hline 1995 & 457 & 72.7 & 47 & 425 & 166 & 61 & 497 & 213 & 57 \\
\hline 1996 & 464 & 71.2 & 51 & 438 & 186 & 58 & 462 & 180 & 61 \\
\hline 1997 & 406 & 72.1 & 46 & 390 & 124 & 68 & 505 & 224 & 56 \\
\hline 1998 & 404 & 71.4 & 44 & 390 & 124 & 68 & 497 & 213 & 57 \\
\hline 1999 & 367 & 72.0 & 47 & 346 & 96 & 72 & 462 & 180 & 61 \\
\hline 2000 & 306 & 71.5 & 51 & 295 & 106 & 64 & 406 & 188 & 54 \\
\hline 2001 & 244 & 71.6 & 47 & 231 & 79 & 66 & 317 & 144 & 55 \\
\hline 2002 & 157 & 70.3 & 52 & 150 & 54 & 64 & 228 & 108 & 53 \\
\hline 2003 & 142 & 69.9 & 49 & 140 & 45 & 68 & 198 & 88 & 56 \\
\hline 2004 & 134 & 68.0 & 46 & 126 & 52 & 59 & - & - & - \\
\hline All years & 3504 & 71.5 & 48 & 3332 & 1168 & 65 & 3013 & 1392 & 54 \\
\hline \multicolumn{10}{|l|}{ Prostaglandins } \\
\hline 1997 & 7 & 74.8 & 43 & 7 & 2 & 71 & 5 & 2 & 60 \\
\hline 1998 & 47 & 74.8 & 46 & 44 & 14 & 68 & 39 & 21 & 46 \\
\hline 1999 & 88 & 72.5 & 32 & 81 & 29 & 64 & 69 & 31 & 55 \\
\hline 2000 & 158 & 75.0 & 40 & 147 & 48 & 67 & 139 & 63 & 55 \\
\hline 2001 & 263 & 74.6 & 43 & 241 & 76 & 68 & 226 & 100 & 56 \\
\hline 2002 & 382 & 72.7 & 48 & 355 & 92 & 74 & 334 & 120 & 64 \\
\hline 2003 & 400 & 73.4 & 44 & 380 & 111 & 71 & 358 & 143 & 60 \\
\hline 2004 & 453 & 73.4 & 51 & 427 & 115 & 73 & - & - & 一 \\
\hline All years & 1798 & 73.4 & 46 & 1682 & 487 & 71 & 1170 & 480 & 59 \\
\hline \multicolumn{10}{|l|}{ All treatments ${ }^{b}$} \\
\hline 1994 & 446 & 71.7 & 45 & 423 & 145 & 66 & 396 & 188 & 53 \\
\hline 1995 & 487 & 71.1 & 46 & 453 & 185 & 59 & 421 & 227 & 46 \\
\hline 1996 & 499 & 71.8 & 46 & 468 & 195 & 58 & 438 & 222 & 49 \\
\hline 1997 & 436 & 71.7 & 48 & 419 & 137 & 67 & 384 & 170 & 56 \\
\hline 1998 & 479 & 71.7 & 49 & 458 & 148 & 68 & 427 & 193 & 55 \\
\hline 1999 & 485 & 71.8 & 47 & 457 & 133 & 71 & 418 & 164 & 61 \\
\hline 2000 & 498 & 72.7 & 51 & 476 & 169 & 64 & 453 & 215 & 53 \\
\hline 2001 & 540 & 73.1 & 48 & 504 & 170 & 66 & 479 & 215 & 55 \\
\hline 2002 & 579 & 71.9 & 52 & 542 & 161 & 70 & 510 & 206 & 60 \\
\hline 2003 & 587 & 72.5 & 49 & 562 & 165 & 71 & 534 & 220 & 59 \\
\hline 2004 & 634 & 72.5 & 54 & 595 & 178 & 70 & - & - & - \\
\hline 1994-1996 & 1432 & 71.8 & 50 & 1344 & 525 & 61 & 1255 & 637 & 49 \\
\hline $1997-2001$ & 2438 & 72.3 & 46 & 2314 & 757 & 67 & 2161 & 957 & 56 \\
\hline 2002-2004 & 1800 & 72.2 & 49 & 1699 & 504 & 70 & 1044 & 426 & 59 \\
\hline All years & 5670 & 72.1 & 48 & 5357 & 1786 & 67 & 4460 & 2020 & 55 \\
\hline
\end{tabular}

$\% \operatorname{trt}=$ percentage of these patients who are persisting with treatment.

$N_{1}=$ number of patients still registered after 1 year, $N_{2}=$ number of patients still registered after 2 years.

${ }^{\text {a }}$ ail $=$ number of these patients with a 90-day period without any therapy for glaucoma.

bIncludes treatment combinations other than the two monotherapies.

estimates (pooled HR of failure $=1.40,95 \%$ CI 1.34, 1.46, Figure 3); this is in close agreement with the estimate from the current study $(1.35,95 \%$ CI $1.21,1.50)$.

However, there was considerable heterogeneity between estimates $(P<0.001)$. The risk of failure was the strongest in a study with relatively fewer participants that used a glaucoma or suspect glaucoma diagnosis as inclusion criteria, ${ }^{24}$ although there was no evidence of small study bias (Begg test $P=0.8$, Egger test $P=0.1$ ). Including change or additions to therapy as failure, attenuated difference in the risk of failure observed between $\beta$-blockers and prostaglandins, in five studies, which looked at it $(1.21,95 \%$ CI 1.17, 1.26). There was little heterogeneity between estimates $(P=0.5)$ and borderline evidence of small study bias (Begg test $P=0.8$, Egger test $P=0.05$ ). Both meta-analyses were dominated 


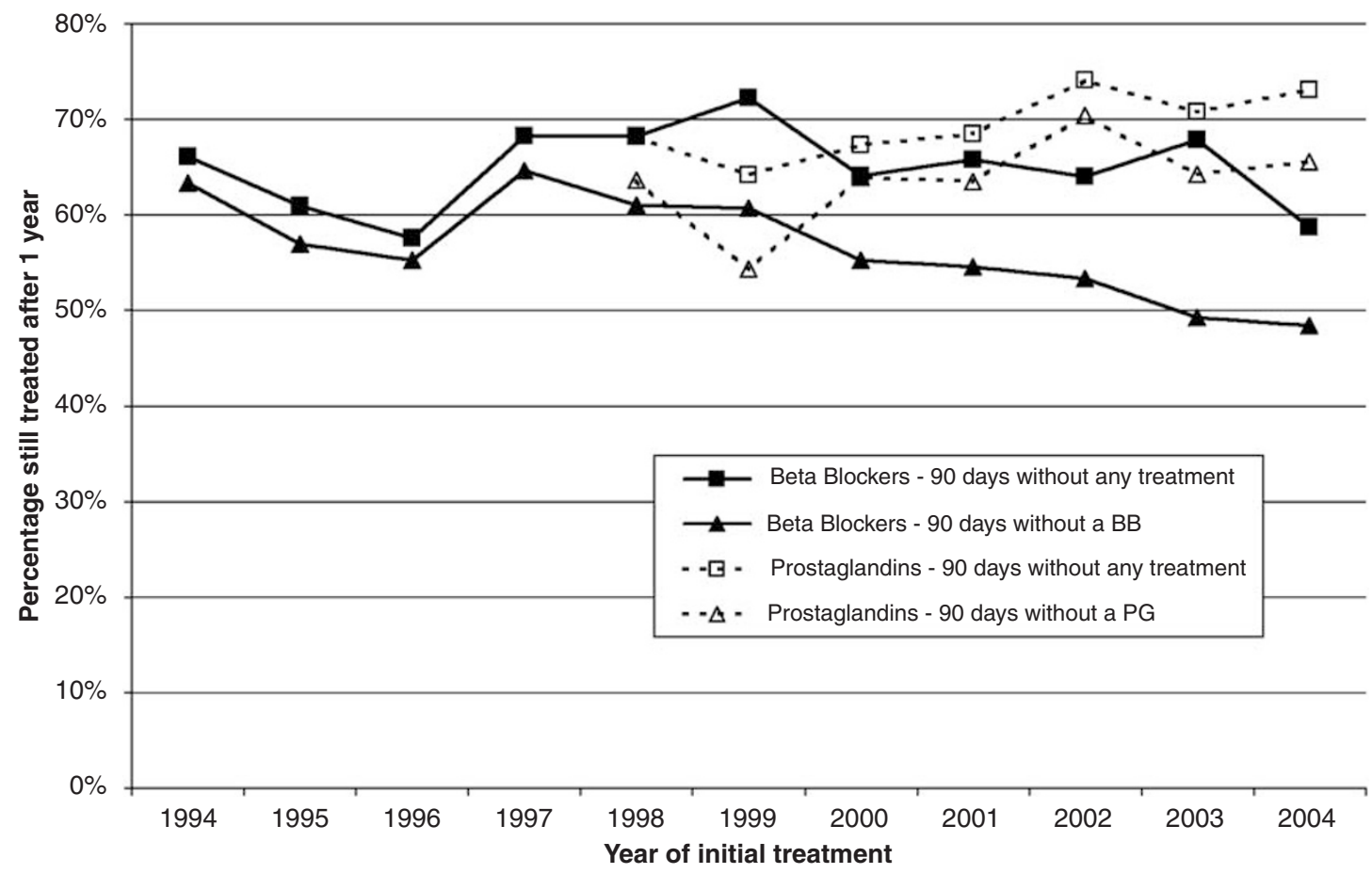

Figure 2 One-year treatment persistence of $\beta$-blockers vs prostaglandins by two failure definitions 1994-2004 (including failure on specific treatment, triangular symbols; and failure on any treatment, square symbols).

Table 2 Proportional hazard models fitting 90-day failure rates $(n=5670)$

\begin{tabular}{|c|c|c|c|c|c|}
\hline & \multirow[t]{2}{*}{$\mathrm{N}$} & \multicolumn{2}{|c|}{ Unadjusted } & \multicolumn{2}{|c|}{ Adjusted $^{\mathrm{a}}$} \\
\hline & & Hazard ratio & $95 \% \mathrm{CI}$ & Hazard ratio & $95 \% C I$ \\
\hline Prostaglandin & 1798 & 1 & - & 1 & - \\
\hline$\beta$-blockers & 3504 & 1.22 & $1.12-1.33$ & 1.05 & $0.93-1.17$ \\
\hline Combination & 368 & 1.37 & $1.17-1.60$ & 1.26 & $1.07-1.48$ \\
\hline Women & 2961 & 1 & - & 1 & - \\
\hline Men & 2709 & 1.04 & $0.97-1.12$ & 1.04 & $0.96-1.12$ \\
\hline Age 35-64 & 1385 & 1.41 & $1.28-1.55$ & 1.43 & $1.30-1.58$ \\
\hline Age $65-74$ & 1615 & 1.04 & $0.94-1.15$ & 1.03 & $0.94-1.14$ \\
\hline Age 75-84 & 1889 & 1 & - & 1 & - \\
\hline Age $85+$ & 781 & 1.24 & $1.10-1.40$ & 1.28 & $1.13-1.44$ \\
\hline Wealthy achievers & 1936 & 1 & - & 1 & - \\
\hline Urban prosperity & 407 & 1.30 & $1.13-1.50$ & 1.16 & $0.96-1.41$ \\
\hline Comfortably off & 1855 & 0.95 & $0.86-1.04$ & 0.93 & $0.84-1.03$ \\
\hline Moderate means & 478 & 1.06 & $0.92-1.22$ & 1.00 & $0.85-1.16$ \\
\hline Hard pressed & 832 & 1.05 & $0.93-1.18$ & 0.99 & $0.88-1.13$ \\
\hline Missing & 162 & 1.11 & $0.89-1.38$ & 1.23 & $0.92-1.64$ \\
\hline
\end{tabular}

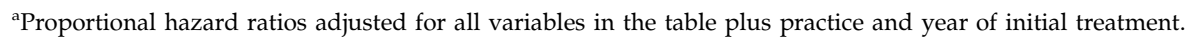

by one study, ${ }_{1}^{18}$ with 57 to $90 \%$ of the statistical weight, respectively. Exclusion of this study had little effect among studies considering failure of initial therapy only (1.43, 95\% CI 1.35, 1.53 after exclusion), but the effect was marginally strengthened after exclusion when studies including changes or additions to therapy were considered $(1.33,95 \%$ CI 1.19, 1.50).

\section{Discussion}

Persistence with initial treatment for glaucoma and ocular hypertension was poor, with a third failing after 1 year, and just under half ( $45 \%$ ) failing by 2 years. This fits with other studies examining persistence with treatment for glaucoma and ocular hypertension, ${ }^{17,30,33}$ as well as 
Table 3 List of studies that have examined persistence with treatment for glaucoma using computerised healthcare data

\begin{tabular}{|c|c|c|c|c|c|c|c|c|c|}
\hline \multirow[t]{2}{*}{ Author } & \multirow[t]{2}{*}{ Population } & \multirow[t]{2}{*}{ Time period } & \multirow[t]{2}{*}{$\begin{array}{l}\text { Inclusion/exclusion } \\
\text { criteria }\end{array}$} & \multirow[t]{2}{*}{ Definition of failure } & \multicolumn{2}{|c|}{$\begin{array}{l}\text { Treatment failure } \\
\text { includes }\end{array}$} & \multirow[t]{2}{*}{$\begin{array}{l}\text { Treatments } \\
\text { compared }(N)\end{array}$} & \multirow[t]{2}{*}{$\begin{array}{l}\text { Relative risk } \\
(95 \% \text { CI })\end{array}$} & \multirow[t]{2}{*}{ Adjustments } \\
\hline & & & & & Stop & $\begin{array}{c}\text { Change/ } \\
\text { add }\end{array}$ & & & \\
\hline \multirow[t]{2}{*}{$\begin{array}{l}\text { Dasgupta } \\
\text { et } a l^{30}\end{array}$} & $\begin{array}{l}\text { Health insurance } \\
\text { claims (Advance PCS } \\
\text { database) from three } \\
\text { different regions in the } \\
\text { United states }\end{array}$ & 1999-2000 & $\begin{array}{l}\text { Less than } 65 \text { years of } \\
\text { age, patients initiated } \\
\text { on one of the seven } \\
\text { treatments for } \\
\text { glaucoma }\end{array}$ & $\begin{array}{l}\geqslant 120 \text { days without } \\
\text { index therapy, switch } \\
\text { or addition in } \\
\text { glaucoma therapy }\end{array}$ & $\sqrt{ }$ & $\sqrt{ }$ & $\begin{array}{l}\text { Latanoprost } \\
\text { (320) vs } \\
\beta \text {-blockers (785) }\end{array}$ & $1.24 *(1.02,1.50)$ & Unadjusted \\
\hline & & & & $\begin{array}{l}\geqslant 120 \text { days without } \\
\text { index therapy }\end{array}$ & $\sqrt{ }$ & $x$ & & $1.63 \dagger(1.26,2.11)$ & Unadjusted \\
\hline \multirow[t]{3}{*}{$\begin{array}{l}\text { Nordstrom } \\
\text { et } a l^{24}\end{array}$} & $\begin{array}{l}\text { UnitedHealthcare } \\
\text { members' ingenix } \\
\text { research database, USA }\end{array}$ & 1995-2001 & $\begin{array}{l}\text { Patients }>30 \text { years } \\
\text { of age, newly } \\
\text { diagnosed with } \\
\text { glaucoma, or } \\
\text { suspect glaucoma. } \\
\text { Excluded if not } \\
\text { continuously } \\
\text { enrolled for } 1 \text { year, } \\
\text { or previous } \\
\text { glaucoma-related } \\
\text { claim }\end{array}$ & $\begin{array}{l}60-120 \text { days } \\
\text { (depending on dosage) } \\
\text { without treatment for } \\
\text { glaucoma, or change in } \\
\text { medication }\end{array}$ & $\sqrt{ }$ & $\times$ & $\begin{array}{l}\text { Prostaglandins } \\
\text { (966) vs } \\
\beta \text {-blockers (1736) }\end{array}$ & $2.50(2.27,2.86)$ & Glaucomatous \\
\hline & & & & & $\sqrt{ }$ & $\times$ & $\begin{array}{l}\text { Prostaglandins } \\
\text { (386) vs } \beta \text { - } \\
\text { blockers (883) }\end{array}$ & $2.27(1.92,2.70)$ & Suspect \\
\hline & & & & & & & & $2.43 \dagger(2.20,2.67)$ & $\begin{array}{l}\text { Combined using } \\
\text { fixed-effects } \\
\text { model } \\
\text { Age, sex, region, } \\
\text { and year of } \\
\text { index date }\end{array}$ \\
\hline Reardon $e t a l^{31}$ & $\begin{array}{l}\text { Insurance claim records } \\
\text { from New England, } \\
\text { USA }\end{array}$ & 1999-2001 & $\begin{array}{l}\text { Patients }>20 \text { years } \\
\text { of age, initiated on } \\
\text { one of } 5 \text { treatments } \\
\text { for glaucoma. } \\
\text { Excluded if not } \\
\text { continuously enrolled, } \\
\text { and/or treatment for } \\
\text { glaucoma } 180 \mathrm{~d} \text { prior } \\
\text { to initiation }\end{array}$ & $\begin{array}{l}120 \text { (one bottle) to } 180 \\
\text { days (more than one } \\
\text { bottle) without index } \\
\text { treatment for } \\
\text { glaucoma, switch or } \\
\text { addition in index }\end{array}$ & $\sqrt{ }$ & $\sqrt{ }$ & $\begin{array}{l}\text { Latanoprost } \\
\text { (683) vs } \\
\text { timolol (1408) }\end{array}$ & $1.36 *(1.19,1.55)$ & Age, sex \\
\hline
\end{tabular}


Table 3 (Continued)

\begin{tabular}{|c|c|c|c|c|c|c|c|c|c|}
\hline \multirow[t]{2}{*}{ Author } & \multirow[t]{2}{*}{ Population } & \multirow[t]{2}{*}{ Time period } & \multirow[t]{2}{*}{$\begin{array}{l}\text { Inclusion/exclusion } \\
\text { criteria }\end{array}$} & \multirow[t]{2}{*}{ Definition of failure } & \multicolumn{2}{|c|}{$\begin{array}{l}\text { Treatment failure } \\
\text { includes }\end{array}$} & \multirow[t]{2}{*}{$\begin{array}{l}\text { Treatments } \\
\text { compared }(N)\end{array}$} & \multirow[t]{2}{*}{$\begin{array}{l}\text { Relative risk } \\
(95 \% \text { CI) }\end{array}$} & \multirow[t]{2}{*}{ Adjustments } \\
\hline & & & & & Stop & $\begin{array}{l}\text { Changel } \\
\text { add }\end{array}$ & & & \\
\hline & & & & $\begin{array}{l}\text { 120-180 d without } \\
\text { index treatment }\end{array}$ & $\sqrt{ }$ & $x$ & & $1.77 \dagger(1.50,2.09)$ & Age, sex \\
\hline \multirow[t]{2}{*}{ Reardon $e t a l^{18}$} & $\begin{array}{l}\text { Protocare Sciences' } \\
\text { managed care } \\
\text { database, USA }\end{array}$ & 1996-2002 & $\begin{array}{l}\text { Patients }>20 \text { years } \\
\text { of age, initiated on } \\
\text { one of the seven } \\
\text { treatments for } \\
\text { glaucoma. Excluded } \\
\text { if initiated on more } \\
\text { than one treatment } \\
\text { for glaucoma, } \\
\text { treatment for } \\
\text { glaucoma } 180 \text { days } \\
\text { before initiation, not } \\
\text { continuously enrolled, } \\
\text { glaucoma-related } \\
\text { surgery }\end{array}$ & $\begin{array}{l}90 \text { days (one bottle) to } \\
180 \text { days (more than } \\
\text { one bottle) without } \\
\text { index treatment for } \\
\text { glaucoma, switch or } \\
\text { addition in OHT } \\
\text { therapy }\end{array}$ & $\sqrt{ }$ & $\sqrt{ }$ & $\begin{array}{l}\text { Latanoprost } \\
\text { (6772) vs timolol } \\
(12298)\end{array}$ & $1.20 *(1.16,1.24)$ & Unadjusted \\
\hline & & & & $\begin{array}{l}\text { 90-180 days without } \\
\text { index treatment for } \\
\text { glaucoma }\end{array}$ & $\sqrt{ }$ & $\times$ & & $1.37 \dagger(1.31,1.42)$ & Unadjusted \\
\hline \multirow[t]{2}{*}{ Schwartz ${ }^{16}$} & $\begin{array}{l}\text { Protocare Sciences' } \\
\text { managed care } \\
\text { database, } \\
\text { USA-subgroup of the } \\
\text { above study }{ }^{18} \text { with } \\
\text { glaucoma diagnosis }\end{array}$ & 1997-2002 & $\begin{array}{l}\text { Patients }>20 \text { years } \\
\text { of age, initiated } \\
\text { on one of the seven } \\
\text { treatments for } \\
\text { glaucoma, with a } \\
\text { glaucoma diagnosis. }\end{array}$ & $\begin{array}{l}90 \text { (one bottle) to } 180 \\
\text { days more than one } \\
\text { bottle) without index } \\
\text { drug, switch or } \\
\text { addition in OHT } \\
\text { therapy }\end{array}$ & $\sqrt{ }$ & $\sqrt{ }$ & $\begin{array}{l}\text { Latanoprost } \\
\text { (583) vs timolol } \\
\text { (891) }\end{array}$ & $1.27(1.13,1.43)$ & Age, sex \\
\hline & & & $\begin{array}{l}\text { Excluded if received } \\
\text { multiple OHT } \\
\text { therapy, or glaucoma } \\
\text { therapy/surgery/ } \\
\text { diagnosis in the year } \\
\text { before initiation }\end{array}$ & $\begin{array}{l}90-180 \text { days without } \\
\text { index drug refill }\end{array}$ & $\sqrt{ }$ & $x$ & & $1.39(1.21,1.58)$ & \\
\hline Shaya et $a l^{32}$ & $\begin{array}{l}\text { Care plan members } \\
\text { of CareFirst BlueCross } \\
\text { BlueShield MD } \\
\text { (Advance PCS } \\
\text { database), USA }\end{array}$ & 1999-2001 & $\begin{array}{l}\text { Patients }>20 \text { and } \\
<65 \text { years of age. } \\
\text { Excluded if treatment } \\
\text { for glaucoma } 180 \text { days } \\
\text { before initiation, or not } \\
\text { continuously enrolled }\end{array}$ & $\begin{array}{l}120 \text { days without index } \\
\text { treatment for glaucoma }\end{array}$ & $\sqrt{ }$ & $\times$ & $\begin{array}{l}\text { Latanoprost } \\
\text { (858) vs timolol } \\
\text { (939) }\end{array}$ & $1.34 \dagger(1.27,1.41)$ & Age, sex \\
\hline
\end{tabular}


Table 3 (Continued)

\begin{tabular}{|c|c|c|c|c|c|c|c|c|c|}
\hline \multirow[t]{2}{*}{ Author } & \multirow[t]{2}{*}{ Population } & \multirow[t]{2}{*}{ Time period } & \multirow[t]{2}{*}{$\begin{array}{l}\text { Inclusion/exclusion } \\
\text { criteria }\end{array}$} & \multirow[t]{2}{*}{ Definition of failure } & \multicolumn{2}{|c|}{$\begin{array}{l}\text { Treatment failure } \\
\quad \text { includes }\end{array}$} & \multirow[t]{2}{*}{$\begin{array}{l}\text { Treatments } \\
\text { compared }(N)\end{array}$} & \multirow[t]{2}{*}{$\begin{array}{l}\text { Relative risk } \\
(95 \% \mathrm{CI})\end{array}$} & \multirow[t]{2}{*}{ Adjustments } \\
\hline & & & & & Stop & $\begin{array}{l}\text { Changel } \\
\text { add }\end{array}$ & & & \\
\hline \multirow[t]{2}{*}{ Spooner et al ${ }^{33}$} & $\begin{array}{l}\text { Health insurers of Blue } \\
\text { Cross CA , USA }\end{array}$ & 1998-1999 & $\begin{array}{l}\text { Patients initiated on } \\
\text { one of the six } \\
\text { treatments for } \\
\text { glaucoma. Excluded } \\
\text { if treatment for } \\
\text { glaucoma } 180 \text { days } \\
\text { before initiation, } \\
\text { or not continuously } \\
\text { enrolled }\end{array}$ & $\begin{array}{l}120 \text { (one bottle) to } 180 \\
\text { days ( } 15 \mathrm{ml} \text { bottle or } \\
\text { more than one bottle) } \\
\text { without refilling the } \\
\text { index drug or changing } \\
\text { therapy }\end{array}$ & $\sqrt{ }$ & $\times$ & $\begin{array}{l}\text { Latanoprost } \\
\text { (242) vs timolol } \\
\text { (547) }\end{array}$ & $1.37 *(1.15,1.64)$ & $\begin{array}{l}\text { Age, sex, } \\
\text { glaucoma type, } \\
\text { and frequency of } \\
\text { visits for } \\
\text { glaucoma and to } \\
\text { ophthalmologist }\end{array}$ \\
\hline & & & & $\begin{array}{l}120-180 \text { days without } \\
\text { refilling the index drug } \\
\text { or changing therapy, or } \\
\text { patients that received } \\
\text { different OHT therapy } \\
\text { regardless of continued } \\
\text { index drug use }\end{array}$ & $\sqrt{ }$ & $\sqrt{ }$ & & $1.61 \dagger(1.31,1.99)$ & \\
\hline \multirow[t]{2}{*}{ Zhou et al ${ }^{17}$} & $\begin{array}{l}\text { General Practice } \\
\text { Research Database of } \\
\text { individual primary } \\
\text { care records, UK }\end{array}$ & 1997-1999 & $\begin{array}{l}\text { Patients given a } \\
\text { glaucoma diagnosis } \\
\text { and initiated on } \\
\text { treatment for } \\
\text { glaucoma }\end{array}$ & $\begin{array}{l}\text { A change in the index } \\
\text { drug (switch or } \\
\text { addition) or referral for } \\
\text { glaucoma surgery }\end{array}$ & $\times$ & $\sqrt{ }$ & $\begin{array}{l}\text { Latanoprost } \\
\text { (149) vs timolol } \\
\text { (632) }\end{array}$ & $1.95(1.60,2.38)$ & $\begin{array}{l}\text { Age, sex, length } \\
\text { of period with } \\
\text { medical records }\end{array}$ \\
\hline & & & & $\begin{array}{l}\text { A change in the index } \\
\text { drug (switch or } \\
\text { addition) or referral for } \\
\text { glaucoma surgery or no } \\
\text { refill of the index drug } \\
\text { for } 60 \text { days }\end{array}$ & $\sqrt{ }$ & $\sqrt{ }$ & & $1.37 *(1.06,1.78)$ & \\
\hline $\begin{array}{l}\text { Five } \\
\text { studies }^{17,18,30,31,33}\end{array}$ & & & & $\begin{array}{l}\text { Failure on index } \\
\text { therapy or change in } \\
\text { glaucoma therapy }\end{array}$ & $\sqrt{ }$ & $\sqrt{ }$ & $\begin{array}{l}\text { Prostaglandins } \\
\text { vs } \beta \text {-blockers }\end{array}$ & $1.21 *(1.17,1.26)$ & $\begin{array}{l}\text { Test for } \\
\text { heterogeneity } \\
P=0.5\end{array}$ \\
\hline $\begin{array}{l}\text { Six } \\
\text { studies }^{18,24,30-33}\end{array}$ & & & & $\begin{array}{l}\text { Failure on index } \\
\text { therapy }\end{array}$ & $\sqrt{ }$ & $\times$ & $\begin{array}{l}\text { Prostaglandins } \\
\text { vs } \beta \text {-blockers }\end{array}$ & $1.40 \dagger(1.34,1.46)$ & $\begin{array}{l}\text { Test for } \\
\text { heterogeneity } \\
P<0.001\end{array}$ \\
\hline
\end{tabular}

The $*, \dagger$ symbols in the table indicate the estimates from each study used in the two meta-analyses. 


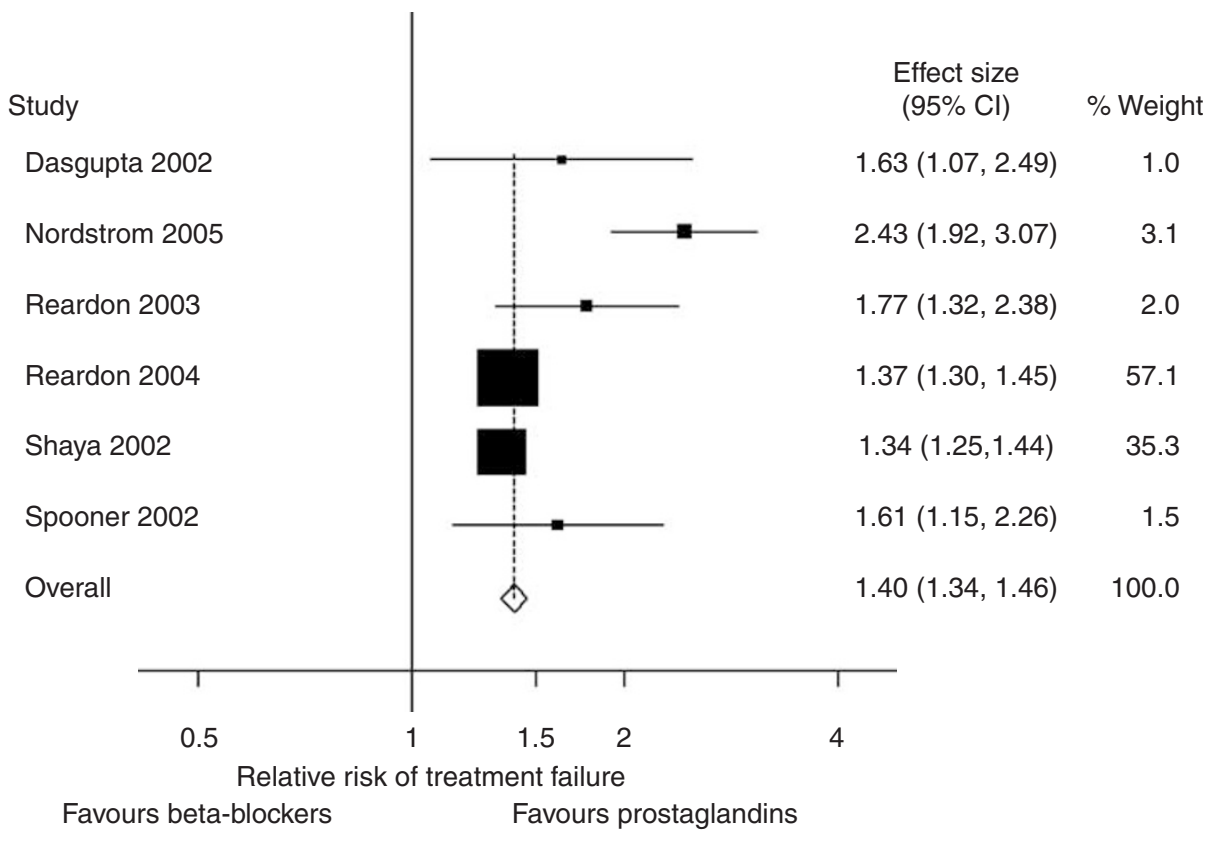

Figure 3 Forest plot showing the relative risk of failure with therapy, comparing $\beta$-blockers with prostaglandins. Box area of each study proportional to the inverse of the variance, with the horizontal lines showing 95\% CI. First author is indicated on the Y-axis. Combined fixed-effects estimate indicated by the dotted vertical line, $95 \%$ CI by diamond.

other chronic conditions in elderly patients, such as hypertension and hypercholesteraemia, where persistence rates are also disappointingly low. ${ }^{34-36}$ Reasons for failure with drug therapy are unclear, and may be related to the presence of other comorbidities, severity of disease, perceived benefit of treatment, drug tolerance, and convenience of use. ${ }^{34,37,38}$ The latter may be of particular relevance in elderly patients using topical treatments for glaucoma where manual dexterity is needed to administer drug therapy.

Failure to persist with ocular hypotensive medications has been shown in both UK and American populations, and has partly been attributed to the modality of treatment. However, the substantial reasons for failure with ocular hypotensive therapy remain largely unknown. Men have previously been shown to be more likely to be treated for glaucoma and ocular hypertension, ${ }^{19,39}$ and data from population-based studies indicate that they are more likely than women to suffer from glaucoma. ${ }^{2}$ Despite this, there does not appear to be any sex difference in persistence with treatment, which agrees with another recent study that showed marginal differences in persistence by gender. ${ }^{24}$ Healthcare databases allow persistence with prescribed drug treatments to be gauged in a large sample, representative of the general population (although they may not contain prescribing data on a small percentage treated privately). ${ }^{21}$ Completeness of recording of prescriptions in the United Kingdom is high and although some errors in recording are inevitable, it is implausible that recording of prescriptions would differ between those prescribed different types of therapy. The main limitation of healthcare databases is that they do not yield information on actual use of medications (ie, adherence to therapy), and contain limited demographic information about the individual, other than sex and age. The DIN-LINK database has both a regional variable and a validated marker of socioeconomic status. ${ }^{23}$ Although those from less privileged circumstances are less likely to be treated for glaucoma ${ }^{19}$ and are more likely to present to health services with more advanced disease than those in more privileged circumstances, ${ }^{40}$ there did not appear to be any socioeconomic differences in persistence with treatment. In agreement with an earlier study, ${ }^{24}$ older age groups were more likely to persist with treatment than younger age groups; although the improvement in persistence tails off in the oldest age group (aged 85 years or more). Age differences in persistence may be influenced by severity of disease. The natural history of glaucoma suggests that younger age groups are more likely to exhibit earlier stages of glaucoma than older patients, but the degree to which this reflects clinical presentation of the disease is unclear. Unfortunately, diagnostic codes for glaucoma in the database do not record severity of disease, with most (78\%), probably for convenience, being recorded as simply having 'glaucoma'. It is possible that the likelihood of surgery for glaucoma may differ by age, which may result in 
short- or long-term discontinuation of drug therapy for certain age groups. The proportion receiving surgery or laser treatment for glaucoma could not be reliably ascertained from the database (less than $1 \%$ of glaucoma related codes were given for glaucomatous surgery). ${ }^{19}$ Hence, this study cannot resolve these issues. However, a previous UK-based study has shown that the percentage receiving surgical interventions for glaucoma is small and diminishing relative to drug therapy in contemporary cohorts. ${ }^{41}$

Numerous studies using healthcare databases have shown superior persistence with prostaglandin treatments than $\beta$-blocker and other medications. However, the degree of benefit varies and appears to depend on the definition of persistence used. A review of studies using different periods to failure but all counting ceasing or changes in treatment as a failure (including data from the current study), consistently found that those on $\beta$-blocker medications were more likely to fail than those on prostaglandins. However, definitions of persistence including changes in treatment may be misleading as this may be driven by the treatment currently in vogue, rather than an inherent failure with treatment. It may also result in a selection bias, where those who swap or add to older treatments may not be comparable to those who switch or add to newer medications. For instance, prostaglandins have been shown to lower IOP more effectively than other classes of medication $^{42,43}$ and may be preferentially given to those with more advanced or aggressive disease. ${ }^{24}$ However, without being able to review medical records or question patients directly, it is not possible to gauge the reasons for switching or adding to therapy, and to ascertain the degree to which this reflects a clinician's assessment of a drug's effectiveness and tolerability or therapeutic fashions. Hence, we believe that the fairest comparison is to only regard cessation of all treatment as a failure. This study is the first to adopt this approach when comparing persistence with $\beta$-blocker and prostaglandin therapy, and this is likely to explain why our results are more modest compared to previous studies. Moreover, we found that $\beta$-blockers were more likely to be given in the short term as an ocular hypotensive in association with topical corticosteroids and perioperative medications, compared to prostaglandin medications, which may reflect different clinical usage of these medications and preference for a less irritable medication post-ocular surgery and/or inflammation. Failure to exclude these individuals (we have excluded them in this study), may act to artificially improve persistence with

prostaglandins (adjusted hazard ratio 1.19, 95\% CI 1.071.33 without exclusion). However, this observation has not been reported in other studies that consider treatment for glaucoma and ocular hypertension without a glaucoma diagnosis. Although the likelihood of failure appeared similar in those prescribed $\beta$-blockers and prostaglandins, patients were more likely to fail on combination therapies. However, only a small percentage of patients $(6 \%)$ are given combination therapies, and these are likely to be a heterogeneous group as medications are varied.

One of the major strengths of this study is that it allows persistence to be examined over a decade (1994-2005) from when $\beta$-blockers were pervasively prescribed to when prostaglandins were introduced (1997 in the United Kingdom) and became the most commonly prescribed ocular hypotensive (as of 2003). We have shown that overall persistence with ocular hypotensive treatment has improved over this period. Whether increased persistence reflects superior effectiveness, tolerance, ${ }^{44}$ and more convenient dosage regimen (ie, with reduced frequency of administration) of newer ocular hypotensives or improvements in management and care of patients remains unclear. Better patient awareness of glaucoma and appreciation among healthcare providers of the need for better compliance with treatment may also partly explain the improvements in persistence observed over time. New approaches to examine persistence with ocular hypotensive therapy, allowing for gaps or restarts in therapy, may well further our understanding of factors influencing persistence with treatment for glaucoma. ${ }^{45,46}$ Recent studies validating the use of pharmacy databases using personal interviews and examination of clinical records may also provide insight into factors affecting adherence with ocular hypotensive medications. ${ }^{47,48}$

\section{Ethics approval}

This study was approved by the National Heath Service Research Ethics Committee for Wandsworth (reference 05/Q0803/162).

\section{Acknowledgements}

We are grateful to CompuFile Limited (Woking, Surrey, UK) who provided technical assistance with DIN-LINK database and for much helpful support. We acknowledge with gratitude the general practitioners who provide data for DIN-LINK.

Contributors: All authors contributed substantially to the conception and design of this paper. CGO drafted the paper and IMC carried out the statistical analysis. The paper was critically appraised by all authors for intellectual content; CGO, and IMC will act as guarantors. The guarantors accept full responsibility for the integrity of the work as a whole. All authors had access to the data and approved the final version to be 
published.This study was funded by a grant from Pfizer Global Pharmaceuticals (Pfizer Limited, Tadworth, Surrey, UK). IMC and SdeW are supported by the BUPA Foundation.

\section{References}

1 Quigley HA, Broman AT. The number of people with glaucoma worldwide in 2010 and 2020. Br J Ophthalmol 2006; 90(3): 262-267.

2 Rudnicka AR, Mt-Isa S, Owen CG, Cook DG, Ashby D. Variations in primary open-angle glaucoma prevalence by age, gender, and race: a Bayesian meta-analysis. Invest Ophthalmol Vis Sci 2006; 47(10): 4254-4261.

3 Resnikoff S, Pascolini D, Mariotti SP, Pokharel GP. Global magnitude of visual impairment caused by uncorrected refractive errors in 2004. Bull World Health Organ 2008; 86(1): 63-70.

4 Heijl A, Leske MC, Bengtsson B, Hyman L, Bengtsson B, Hussein M. Reduction of intraocular pressure and glaucoma progression: results from the Early Manifest Glaucoma Trial. Arch Ophthalmol 2002; 120(10): 1268-1279.

5 Stewart WC, Chorak RP, Hunt HH, Sethuraman G. Factors associated with visual loss in patients with advanced glaucomatous changes in the optic nerve head. Am J Ophthalmol 1993; 116(2): 176-181.

6 Tsai JC, McClure CA, Ramos SE, Schlundt DG, Pichert JW. Compliance barriers in glaucoma: a systematic classification. J Glaucoma 2003; 12(5): 393-398.

7 Schwartz GF. Compliance and persistency in glaucoma followup treatment. Curr Opin Ophthalmol 2005; 16(2): 114-121.

8 Schuman JS. Antiglaucoma medications: a review of safety and tolerability issues related to their use. Clin Ther 2000; 22(2): 167-208.

9 Kirwan JF, Nightingale JA, Bunce C, Wormald R. Beta-blockers for glaucoma and excess risk of airways obstruction: population based cohort study. BMJ 2002; 325(7377): 1396-1397.

10 Gandolfi SA, Chetta A, Cimino L, Mora P, Sangermani C, Tardini MG. Bronchial reactivity in healthy individuals undergoing long-term topical treatment with beta-blockers. Arch Ophthalmol 2005; 123(1): 35-38.

11 Krousel-Wood M, Thomas S, Muntner P, Morisky D. Medication adherence: a key factor in achieving blood pressure control and good clinical outcomes in hypertensive patients. Curr Opin Cardiol 2004; 19(4): 357-362.

12 Claxton AJ, Cramer J, Pierce C. A systematic review of the associations between dose regimens and medication compliance. Clin Ther 2001; 23(8): 1296-1310.

13 Kass MA, Gordon M, Meltzer DW. Can ophthalmologists correctly identify patients defaulting from pilocarpine therapy? Am J Ophthalmol 1986; 101(5): 524-530.

14 Robin AL, Novack GD, Covert DW, Crockett RS, Marcic TS. Adherence in glaucoma: objective measurements of oncedaily and adjunctive medication use. Am J Ophthalmol 2007; 144(4): 533-540.

15 Wilensky J, Fiscella RG, Carlson AM, Morris LS, Walt J. Measurement of persistence and adherence to regimens of IOP-lowering glaucoma medications using pharmacy claims data. Am J Ophthalmol 2006; 141(1 Suppl): S28-S33.

16 Schwartz GF, Reardon G, Mozaffari E. Persistency with latanoprost or timolol in primary open-angle glaucoma suspects. Am J Ophthalmol 2004; 137(1 Suppl): S13-S16.
17 Zhou Z, Althin R, Sforzolini BS, Dhawan R. Persistency and treatment failure in newly diagnosed open angle glaucoma patients in the United Kingdom. Br J Ophthalmol 2004; 88(11): 1391-1394.

18 Reardon G, Schwartz GF, Mozaffari E. Patient persistency with topical ocular hypotensive therapy in a managed care population. Am J Ophthalmol 2004; 137(1 Suppl): S3-12.

19 Owen CG, Carey IM, De WS, Whincup PH, Wormald R, Cook DG. The epidemiology of medical treatment for glaucoma and ocular hypertension in the United Kingdom: 1994 to 2003. Br J Ophthalmol 2006; 90(7): 861-868.

20 Carey IM, Cook DG, De WS, Bremner SA, Richards N, Caine $\mathrm{S}$ et al. Developing a large electronic primary care database (Doctor's Independent Network) for research. Int J Med Inform 2004; 73(5): 443-453.

21 Carey IM, Cook DG, De Wilde S, Bremner SA, Richards N, Caine $S$ et al. Implications of the problem orientated medical record (POMR) for research using electronic GP databases: a comparison of the Doctors Independent Network Database (DIN) and the General Practice Research Database (GPRD). BMC Fam Pract 2003; 4(1): 14.

22 De Wilde S, Carey IM, Bremner SA, Richards N, Hilton SR, Strachan DP et al. A comparison of the recording of 30 common childhood conditions in the Doctor's Independent Network and General Practice Research Databases. Health Stat Q 2004; 22): 21-31.

23 CACI. ACORN 2003 User Guide 2004. http:/ / www.caci. co.uk/pdfs/new\%20acorn\%20brochure.pdf. (Accessed December 2007).

24 Nordstrom BL, Friedman DS, Mozaffari E, Quigley HA, Walker AM. Persistence and adherence with topical glaucoma therapy. Am J Ophthalmol 2005; 140(4): 598-606.

25 Cox DR. Regression models and life tables. J R Stat Soc $B$ 1972; 34: 187-220.

26 Deeks JJ, Altman DG, Bradburn MJ. Statistical methods for examining heterogeneity and combining results from several studies in meta-analysis. In: Egger M, Davey Smith G, Altman DG (eds). Systemative Reviews in Health Care: Meta-Analysis in Context, 2nd ed BMJ Books: London, 2001, pp 285-312.

27 Egger M, Davey Smith G, Schneider M, Minder C. Bias in meta-analysis detected by a simple, graphical test. BMJ 1997; 315(7109): 629-634.

28 Light RJ, Pillemar DB. Summing Up: The Science of Reviewing Research. Harvard University Press: Cambridge, MA, USA, 1984.

29 Begg CB, Mazumdar M. Operating characteristics of a rank correlation test for publication bias. Biometrics 1994; 50(4): 1088-1101.

30 Dasgupta S, Oates V, Bookhart BK, Vaziri B, Schwartz GF, Mozaffari E. Population-based persistency rates for topical glaucoma medications measured with pharmacy claims data. Am J Manag Care 2002; 8(10 Suppl): S255-S261.

31 Reardon G, Schwartz GF, Mozaffari E. Patient persistency with pharmacotherapy in the management of glaucoma. Eur J Ophthalmol 2003; 13(Suppl 4): S44-S52.

32 Shaya FT, Mullins CD, Wong W, Cho J. Discontinuation rates of topical glaucoma medications in a managed care population. Am J Manag Care 2002; 8(10 Suppl): S271-S277.

33 Spooner JJ, Bullano MF, Ikeda LI, Cockerham TR, Waugh WJ, Johnson T et al. Rates of discontinuation and change of glaucoma therapy in a managed care setting. Am J Manag Care 2002; 8(10 Suppl): S262-S270. 
34 Osterberg L, Blaschke T. Adherence to medication. N Engl J Med 2005; 353(5): 487-497.

35 Benner JS, Glynn RJ, Mogun H, Neumann PJ, Weinstein $\mathrm{MC}$, Avorn J. Long-term persistence in use of statin therapy in elderly patients. JAMA 2002; 288(4): 455-461.

36 Chapman RH, Benner JS, Petrilla AA, Tierce JC, Collins SR, Battleman DS et al. Predictors of adherence with antihypertensive and lipid-lowering therapy. Arch Intern Med 2005; 165(10): 1147-1152.

37 DiMatteo MR, Haskard KB, Williams SL. Health beliefs, disease severity, and patient adherence: a meta-analysis. Med Care 2007; 45(6): 521-528.

38 DiMatteo MR, Giordani PJ, Lepper HS, Croghan TW. Patient adherence and medical treatment outcomes: a metaanalysis. Med Care 2002; 40(9): 794-811.

39 Friedman DS, Nordstrom B, Mozaffari E, Quigley HA. Variations in treatment among adult-onset open-angle glaucoma patients. Ophthalmology 2005; 112(9): 1494-1499.

40 Fraser S, Bunce C, Wormald R, Brunner E. Deprivation and late presentation of glaucoma: case-control study. BMJ 2001; 322(7287): 639-643.

41 Bateman DN, Clark R, Azuara-Blanco A, Bain M, Forrest J. The impact of new drugs on management of glaucoma in Scotland: observational study. BMJ 2001; 323(7326): 1401-1402.

42 Hedman K, Alm A, Gross RL. Pooled-data analysis of three randomized, double-masked, six-month studies comparing intraocular pressure-reducing effects of latanoprost and timolol in patients with ocular hypertension. J Glaucoma 2003; 12(6): 463-465.

43 Cohen JS, Gross RL, Cheetham JK, VanDenburgh AM, Bernstein P, Whitcup SM. Two-year double-masked comparison of bimatoprost with timolol in patients with glaucoma or ocular hypertension. Surv Ophthalmol 2004; 49(Suppl 1): S45-S52.

44 Schwartz GF, Platt R. Measuring persistency and intraocular pressure-controlled days in patients receiving topical glaucoma medications. Am J Manag Care 2002; 8(10 Suppl): S278-S280.

45 Lee PP, Walt JG, Chiang TH, Guckian A, Keener J. A gap analysis approach to assess patient persistence with glaucoma medication. Am J Ophthalmol 2007; 144(4): 520-524.

46 Schwartz GF, Platt R, Reardon G, Mychaskiw MA. Accounting for restart rates in evaluating persistence with ocular hypotensives. Ophthalmology 2007; 114(4): 648-652.

47 Friedman DS, Quigley HA, Gelb L, Tan J, Margolis J, Shah $\mathrm{SN}$ et al. Using pharmacy claims data to study adherence to glaucoma medications: methodology and findings of the Glaucoma Adherence and Persistency Study (GAPS). Invest Ophthalmol Vis Sci 2007; 48(11): 5052-5057.

48 Quigley HA, Friedman DS, Hahn SR. Evaluation of practice patterns for the care of open-angle glaucoma compared with claims data: the Glaucoma Adherence and Persistency Study. Ophthalmology 2007; 114(9): 1599-1606. 